\title{
Factores del entorno que influyen en la adopción de e-procurement en empresas de la industria del mueble en España
}

\section{Influence of environmental factors on e-procurement adoption process in furniture industry in Spain}

\author{
Alba Soraya Aguilar-Jiménez \\ PhD. Integración de las Tecnologías de Información en las \\ Organizaciones, Universidad Politécnica de Valencia (España) \\ Docente Tiempo Completo, Investigadora Grupo GEETIC, \\ Universidad Pontificia Bolivariana \\ Bucaramanga, Colombia \\ alba.aguilar@upb.edu.co
}

\begin{abstract}
Resumen- Este estudio examina los factores del entorno empresarial, asociados con la adopción de innovaciones TIC para el aprovisionamiento electrónico en pequeñas y medianas empresas. La comprobación empírica se llevó a cabo a partir de información suministrada por e-business w@tch de un grupo de 120 empresas del sector del mueble en España. Se empleó en el análisis de asociación por medio de Tablas de contingencia para encontrar relación estadísticamente significativa entre la presión externa recibida de los proveedores para adoptar determinadas TIC y la ubicación geográfica de los principales proveedores con el nivel de adopción de innovaciones TIC para el aprovisionamiento.
\end{abstract}

Palabras clave- Adopción de innovaciones, tecnologías de información y comunicaciones, influencia de proveedores, pyme, industria del mueble, aprovisionamiento electrónico.

Abstract- This study examines external factors, associated with the adoption of ICT innovations for electronic procurement in SMEs. The empirical test was conducted based on information supplied by e-business w@ tch survey on a group of $\mathbf{1 2 0}$ companies in the furniture industry in Spain. It was used in association analysis, basing on contingency tables that found statistically significant relationship between pressure received from external suppliers to adopt ICT, and the geographic location of the main suppliers with the level of adoption of ICT innovations for procurement.

Keywords- Adoption of innovations, information and communications technologies, suppliers influence, SME, furniture industry, e-procurement.

\section{INTRODUCCIÓN}

Tradicionalmente el proceso de compras se ha limitado al uso del teléfono, correo electróni- co, fax o EDI (Intercambio electrónico de datos Electronic Data Interchange), pero las compras a través de Internet han abierto un nuevo mundo a empresas y proveedores, que ofrecen numerosas herramientas que ayudan a realizar todo tipo de actividades de aprovisionamiento.

El concepto de "e-procurement" (aprovisionamiento electrónico), entendido como la realización de actividades operativas y estratégicas asociadas a los procesos de aprovisionamiento en las empresas y sus relaciones con proveedores, empleando para ello Tecnologías de información y comunicación (en adelante TIC) e Internet [1], está cobrando fuerza en empresas de diferentes tamaños. Las organizaciones, cada vez, dan más importancia al uso de TIC e Internet en el proceso de compras pues encuentran una cantidad enorme de beneficios potenciales ya que permiten mejorar los flujos de información entre empresas y, a su vez, aumentar la cooperación y disminuir los costes y el tiempo de las transacciones y se mejora con ello las relaciones entre proveedores y clientes [2].

Las ventajas más conocidas del uso del aprovisionamiento electrónico apuntan a la reducción de tiempos, costes y carga administrativa, así como a la mejora de las relaciones entre proveedores y clientes y conformación de redes, e incluso hay quienes aseguran que tiene una influencia directa en la mejora de resultados financieros [2], [3], [4], [5], [6], [7], [8], [9], [10] 
Sin embargo, si bien las ventajas de la adopción del e-procurement (en adelante se utilizará como sinónimo de aprovisionamiento electrónico) son más que conocidas, no todas las empresas pueden esperar obtener los mismos beneficios ya que estos pueden ser de diferentes tipos y depender de múltiples factores $\mathrm{y}$, por tanto, el proceso de adopción de las TIC debe estar acorde a sus condiciones particulares y a las exigencias del entorno.

Pequeñas y medianas empresas cada vez son más conscientes de la importancia de invertir en TIC, más aún si estas inversiones redundan en beneficios tangibles [11]. Para el caso del aprovisionamiento electrónico, si bien se han identificado beneficios estratégicos, operacionales y de oportunidad [6], siguen existiendo barreras que inhiben este proceso de adopción [12], [13], [14], e incluso hay estudios que resaltan la existencia de inhibidores del uso de las TIC en diferentes contextos empresariales [12], [13], [15], [16], [17], [18].

Algunos de los autores [19], [20], [21], [22], [23], [24], consideran que la adopción de TIC sigue el proceso de cualquier otra innovación dentro de una empresa. Este proceso de difusión de innovaciones, entendidas como las prácticas u objetos percibidos como nuevos por un individuo o grupo de individuos, ha sido ampliamente estudiado por Everett Rogers [25] y busca explicar la manera como las personas, los grupos, las organizaciones, comunidades y sociedades adoptan una innovación de cualquier tipo. El proceso de difusión de innovaciones sigue una curva en forma de "S" en función de la velocidad de adopción y el número de adoptantes, y explica claramente cómo nuevas ideas y productos se distribuyen, y por qué otros muy buenos no logran hacerlo o no permanecen el tiempo necesario para tener éxito [25]. De acuerdo con este planteamiento, los recursos de TIC son herramientas que apoyan el proceso de transformación organizacional, y son considerados innovaciones que deben ser difundidas gradualmente en las empresas al pasar por unas etapas predefinidas; lo que hace que estas propuestas evolutivas consideren todo tipo de tecnologías, desde hardware, hasta software, bases de datos y redes; como parte del proceso. Este planteamiento se condensa de manera explícita en el "modelo teórico para la adopción de innova- ciones TIC para el aprovisionamiento empresarial" propuesto por Aguilar-Jiménez [1], y se emplea como base para analizar el proceso de adopción del e-procurement en el presente estudio.

Este fenómeno ha sido ampliamente estudiado desde diversas perspectivas, contextos y modelos teóricos [19], [20], [22], [23], [26], [27], [28], que permite identificar aspectos comunes que influyen en la toma de decisiones en relación a la adopción de innovaciones de TIC. En España, las pequeñas y medianas empresas $\left(\right.$ pymes $^{1}$ ) cada vez tienen mayor acceso a las TIC y muestran un notable interés hacia su incorporación, sin embargo, es evidente que la adopción de innovaciones TIC para soportar procesos de aprovisionamiento es un proceso progresivo en el que influyen diversos aspectos. Este grupo de empresas no sólo tienen características diferentes entre sí, sino necesidades específicas y disponibilidad de recursos generalmente limitada, razón por la cual el análisis de los aspectos que influyen adopción de innovaciones de TIC en las empresas para soportar actividades de aprovisionamiento, debe estar adaptado a sus condiciones particulares.

Este análisis es un tema que ha Ilamado la atención de diferentes autores en todo el mundo, quienes han estudiado este proceso en diferentes contextos [4], [6], [7], [14]. Este interés particular se refleja en estudios tempranos realizados principalmente en Estados Unidos, como es el caso del análisis de la adopción de EDI realizado por lacovou et al. [29] o de factores de influencia en el aprovisionamiento electrónico realizado por Heckman [30], pero que, cada vez más, despiertan el interés científico como lo demuestran los estudios de los últimos años ya no sólo en Estados Unidos sino en países de Europa y Asia [14], [31], [32], [33], [34], [35], [36], [37], [38], [39], [40], [41], [42], [43], [44]. Estos estudios en general evidencian de una parte la existencia de aspectos de diversos tipos que influyen en que las empresas tomen la decisión de invertir en TIC, y de otra parte el hecho de que empresas de todos los sectores,

1 El acrónimo PYME (Pequeña y Mediana Empresa) ha sufrido un proceso de lexicalización que lo ha convertido en un nombre común. La última edición del Diccionario de la RAE la califica como sustantivo femenino con el significado de "empresa mercantil, industrial, etc., compuesta por un número reducido de trabajadores, y con un moderado volumen de facturación". El plural de este sustantivo es pymes. www.rae.es. Septiembre 30 de 2010. 
tamaños y países cada vez son más conscientes de la importancia del uso de estas TIC en sus actividades y comunicaciones diarias, así como de formar parte de redes virtuales. Sin embargo, pese a ser un tópico ampliamente estudiado a nivel internacional, en España todavía hay mucho por explorar, no sólo a nivel de la adopción de innovaciones de TIC en el aprovisionamiento empresarial, sino enmarcado en la realidad de la pyme española de sectores industriales específicos.

En este orden de ideas, el presente trabajo busca identificar, a nivel teórico y con ayuda de fuentes de información secundarias, los aspectos del entorno empresarial que inciden en el proceso de adopción de innovaciones TIC en actividades de aprovisionamiento, en pequeñas y medianas empresas en España del sector de fabricación del mueble.

\section{LAS TIC EN EL APROVISIONAMIENTO EMPRESARIAL DE PYMES}

Desde la irrupción de Internet, las pymes como el resto de empresas, se han visto inmersas en una nueva realidad en la que el uso de TIC juega un papel preponderante en el posicionamiento en los mercados, con sus consecuentes efectos en la competitividad y rentabilidad esperada, situación que las ha llevado a desarrollar nuevos modelos de negocio basados en estrategias fundamentadas en torno a ineludibles inversiones en tecnología. El fenómeno de las TIC en la evolución del negocio electrónico especialmente en grandes empresas norteamericanas y europeas ha sido ampliamente tratado por diversos autores [45], [46], [47], [48] y, aunque no son muchos los que han adaptado sus modelos de investigación a condiciones particulares, por ejemplo, a las pymes, sectores industriales o ámbitos territoriales específicos, no por ello se puede negar o afirmar que sus resultados sean válidos en cualquier contexto empresarial analizado.

Según Grandon y Pearson [49], los beneficios del comercio electrónico no son solamente para las grandes empresas, las pymes pueden también beneficiarse de él, adicionalmente creen que su adopción puede "nivelar el campo de juego" con las grandes empresas, para proporcionar independencia espacial y temporal y facilitar la comunicación [49]. En la actualidad las Pymes han descubierto que pueden utilizar el formato universal de la tecnología de Internet para obtener muchos de los beneficios asociados con el intercambio electrónico de datos con clientes y proveedores, sin necesidad de realizar costosas inversiones en equipos informáticos, software y asistencia técnica especializada [48], lo que vincula cada vez más a las pymes al mundo del negocio electrónico.

En lo que se refiere a la gestión de la cadena de suministro, Internet sirve de soporte al desarrollo conjunto de un producto, pasando por la identificación y desarrollo de los proveedores o e-procurement, la gestión de la demanda, logística y en general de los diversos procesos y sistemas empresariales [50]. Sin embargo, si bien el uso de Internet en la gestión de compras se ha desarrollado rápidamente al crear una gran variedad de aplicaciones para apoyar los procesos de aprovisionamiento en la empresa [51], el e-procurement no ha sido suficientemente difundido y adoptado de manera homogénea en empresas de diferentes tamaños y sectores industriales. Las empresas españolas, y en especial las Pymes han reaccionado a las alteraciones del entorno asociadas al uso de TIC en forma desigual entre ellas, situándolas en desventaja en cuanto a los efectos de su uso en la competitividad [23].

El uso de las TIC y en especial de Internet en actividades de aprovisionamiento ha desencadenado una revolución importante en las relaciones entre empresas (Business to Business - B2B) y proveedores, sin embargo el grado de asimilación de dichas tecnologías aún es incipiente especialmente en pymes españolas, las cuales en muchos casos aún realizan sus compras por métodos tradicionales o se encuentran en etapas tempranas de adopción [5], [8], [23], [52], [53], [54]. La dinámica de adopción de TIC en el negocio electrónico ha sido caracterizada como un proceso evolutivo por el que las empresas deben pasar de manera gradual y que usualmente van desde niveles iniciales asociados con un primer acercamiento de la empresa a los recursos ofrecidos por Internet, hasta niveles avanzados de transformación organizacional basada en la red [27], [53], [55], [56], [57].

Para Rogers [25], el éxito de la adopción de una tecnología se encuentra en la naturaleza misma de la innovación, y define la innovación 
como una idea, práctica u objeto que es percibido como nuevo por un individuo o unidad de adopción. Así pues, este proceso de adopción de innovaciones TIC implica una transformación empresarial que obliga a las pymes a recorrer un camino que las conduzca a la adopción paulatina de innovaciones tecnológicas; proceso cuyo éxito depende de factores internos y externos a la empresa, así como de una adecuada planeación y ejecución de las inversiones en TIC, de sus características y su estrategia de negocio electrónico particular.

\section{MODELO DE INVESTIGACIÓN E HIPÓTESIS}

Teniendo en cuenta que no es posible incluir todos los aspectos potenciales que afectan la adopción de innovaciones TIC en actividades de aprovisionamiento electrónico en las empresas, la selección de los aspectos se basó en una exhaustiva revisión teórica tanto del uso de TIC y la comprensión de las actividades asociadas al proceso de aprovisionamiento electrónico, como del proceso de adopción de innovaciones de TIC y el tamaño de las empresas. En consecuencia, este estudio pretende analizar el efecto de aspectos del entorno en el proceso de adopción de innovaciones TIC en actividades de aprovisionamiento electrónico en pymes españolas.

Según el marco TOE (Technology-OrganizationEnvironment framework) [58], los factores que afectan la toma de decisiones de organización en materia de innovación tecnológica se pueden agrupar en tres bloques contextuales que son: el tecnológico, el organizacional y el entorno, los cuales están relacionados con la intención de una empresa de adoptar o no una nueva tecnología, así como el impacto en el rendimiento en relación con la asimilación de las TIC en sus procesos de negocio.

Según Tornatzky y Fleischer [58], en el entorno empresarial existen factores que pueden influir en el proceso de toma de decisiones asociadas con las TIC, los cuales están relacionados con las características de la industria, la disponibilidad y coste de personal capacitado, cambios en la demanda del mercado, presión de parte de competidores innovadores o de las relaciones clienteproveedor, presión del mercado o de la industria, acceso a proveedores de servicios tecnológicos, relaciones gubernamentales, normas y reglamentos, por mencionar algunos ejemplos.

En consecuencia, el modelo de investigación que se presenta en la Fig. 1, sugiere la existencia de aspectos del entorno que están relacionados con el proceso de adopción de innovaciones TIC en el aprovisionamiento empresarial, entendiendo este proceso dentro del concepto de "rapidez de adopción de innovaciones (innovativeness)" de la teoría de difusión de innovaciones de Rogers [25].

Fig. 1. MODELO TEÓRICO PARA LA INVESTIGACIÓN EN RELACIÓN A LOS ASPECTOS DEL ENTORNO EMPRESARIAL



Fuente: Autor del proyecto basado en Aguilar-Jiménez [1]

Es decir, la decisión de una empresa de adoptar o no una nueva tecnología y, para el caso particular, de adoptar o no innovaciones de TIC para soportar los procesos de aprovisionamiento, puede ser afectada por aspectos del entorno. En consecuencia, y a partir del marco TOE [58], se configuran las hipótesis de investigación asociadas a cada uno de estos aspectos.

Como puede apreciarse en la Fig. 1, dentro del contexto externo se pueden identificar aspectos determinantes para la actividad innovadora lo que lleva a identificar variables y plantear hipótesis desde una perspectiva teórica y de acuerdo al entorno particular asociado al aprovisionamiento en las Pymes.

\section{A. Variable "Nivel de adopción de innovaciones de TIC en el aprovisionamiento empresarial"}

La variable dependiente Nivel de adopción de innovaciones de TIC en el aprovisionamiento empresarial fue definida según el modelo propuesto en Aguilar-Jiménez que fue desarrollado específicamente para analizar "la adopción de innovaciones TIC para el aprovisionamiento empresarial o 
e-procurement" [1]. Este modelo plantea la existencia de tres niveles que permiten categorizar las empresas según su nivel de adopción TIC para soportar actividades de aprovisionamiento y cada uno de estos niveles está asociado a una determinada combinación de aspectos asociados a las actividades basadas en TIC que realizan las empresas para aprovisionarse, y que determinan el momento evolutivo en que se encuentra una organización [1].

Las empresas clasificadas como Pioneras son aquellas que ya han adoptado y emplean las TIC para realizar actividades de aprovisionamiento empresarial.

Las Seguidoras, pese al probable escepticismo inicial, una vez conocidos los beneficios de las TIC en el aprovisionamiento muestran interés por aceptar y emplear las posibilidades ofrecidas en sus procesos de compra y han empezado a emplearlas.

Finalmente, las Rezagadas pueden haber realizado pequeñas inversiones TIC, son muy escépticas a las innovaciones por lo que no presentan perspectivas reales cercanas para emplear las TIC en sus procesos de aprovisionamiento, ya que no las consideran necesarias para permanecer en los mercados [1].

\section{B. Variable el subsector industrial al que perte- nece la empresa}

Un primer aspecto que puede ejercer influencia en las decisiones TIC de las empresas es el hecho de pertenecer a determinada industria, especialmente en el contexto de las Pymes, dado que muchas veces estas empresas tienden a tener menos recursos para aumentar su base de conocimiento interno sobre las actuales tendencias tecnológicas y su evolución, por lo que tienden a dejarse influenciar por la dinámica de las empresas del sector y confiar en sus contrapartes para su propia toma de decisiones[15].

En el contexto de la adopción de las TIC, el efecto de la industria es considerado por algunos autores como variable de control [14], [39], [59], mientras que otros la consideran un aspecto del entorno, pero en el sentido de la presión que pueden recibir las empresas de clientes o proveedores [60]. El marco teórico TOE por su parte considera las características de la industria, junto con la estructura del mercado como un aspecto del entorno [58].

El efecto de la industria no sólo ha sido estudiado desde el punto de vista de los aspectos influyentes, sino desde el punto de vista del proceso de adopción de las TIC. Daniel et al.[56] consideran que el hecho de pertenecer a una etapa u otra de adopción del comercio electrónico depende de aspectos contextuales como la industria a la que pertenece la empresa.

Para el caso particular del presente estudio, el efecto de la industria como tal no se puede probar en sentido estricto, dado que las empresas objeto de estudio pertenecen a un mismo sector industrial. Esto mismo sucede en el estudio realizado por Zhu [46], quien analiza el efecto del subsector industrial en el valor de las capacidades de comercio electrónico y la infraestructura TIC en las empresas del sector de comercio minorista. Así pues, el éxito en el uso de las TIC en el sector, no depende solamente de las iniciativas de las empresas fabricantes de muebles, sino que está directamente relacionado con los avances en materia de e-commerce en proveedores y comerciantes.

En consecuencia, dado que el modelo fue elaborado para un sector industrial específico, como es el sector del mueble, el efecto del subsector al que pertenece una empresa no se tendrá en cuenta.

\section{Variable ubicación geográfica de proveedores}

Dentro del contexto del entorno, otro aspecto que ha sido estudiado por diversos autores en el área de la adopción de las TIC, es la distancia geográfica de los mercados. Con el uso de las TIC las empresas más pequeñas pueden llegar a alcanzar mercados más amplios y operar internacionalmente incluso con recursos limitados [24]. Diversos autores sugieren la importancia del uso de las TIC en la mejora de relaciones con clientes y consideran el alcance global de la empresa y el mercado objetivo de la empresa como aspecto de influencia en la toma de decisiones TIC [39], [61], [62].

Si bien es cierto que el uso de medios electrónicos en las transacciones comerciales reducen las barreras comerciales relacionadas con la distancia, según varios estudios, la distancia parece 
ser aún un importante obstáculo al comercio electrónico [63].

Asimilado este concepto al caso del aprovisionamiento electrónico y habida cuenta que el uso de las TIC favorece las relaciones con clientes y proveedores, parece claro que si la distancia y ubicación geográfica de los clientes es un aspecto de influencia para la adopción del comercio electrónico, por analogía, en el caso del aprovisionamiento electrónico probablemente se pueda esperar el mismo efecto si se analiza la ubicación geográfica de los proveedores.

Si bien la ubicación geográfica de proveedores no es una variable que aparezca en estudios teóricos anteriores en el sentido estricto, si es un aspecto importante en relación con el proceso de aprovisionamiento empresarial y debe tenerse en cuenta en este análisis. Es decir, aunque la ubicación geográfica de proveedores no es analizada como aspecto de influencia en el comercio electrónico con proveedores, parece interesante tenerlo en cuenta como una adaptación del efecto de la ubicación geográfica del mercado en la adopción del comercio electrónico con clientes, de lo cual si existen evidencias teóricas.

En este sentido consideraremos que su efecto de la ubicación geográfica de proveedores en la adopción de innovaciones TIC para el aprovisionamiento se puede asimilar al efecto de la ubicación geográfica de clientes en el comercio electrónico, lo que da lugar a la hipótesis de trabajo:

Hipótesis: La ubicación geográfica de los proveedores está relacionada con el nivel de adopción de innovaciones de TIC para apoyar actividades de aprovisionamiento empresarial.

\section{Variable presión externa recibida de los pro- veedores para adoptar determinadas TIC}

A partir de la revisión teórica se puede extraer que una de las principales razones para que las empresas, en especial las Pymes, adopten TIC tales como EDI, es la presión recibida de parte de sus socios comerciales [29], [49], especialmente si existen relaciones de dependencia con los proveedores o si estos tienen alto poder de negociación [64], [65], varios autores consideran la presión externa como un aspecto de potencial influencia en la adopción de innovaciones de las TIC [14], [17], [31], [39], [61], [63], [66], [67], [68], [69], [70].
Esta presión recibida por parte de los socios comerciales en algunos casos es entendida como presión competitiva [24], [49], o efecto de la industria [60], e involucra factores relacionados con la competencia, el entorno social, la dependencia de otras empresas que usan comercio electrónico, la industria o incluso el gobierno [49]. Para el caso particular del aprovisionamiento electrónico, esta variable es entendida como el grado de presión de parte de proveedores, para que una empresa adopte una nueva tecnología.

Este aspecto parece ser particularmente importante en las relaciones empresa-proveedor, ya que su efecto en la adopción las TIC ha resultado ser significativo en estudios anteriores [14], [39], [64], por lo que se considera pertinente proponer una hipótesis que recoja este fenómeno, la cual se define como:

Hipótesis: Las empresas que han experimentado presión de sus proveedores para adaptar soluciones TIC, tienden a estar más avanzadas en términos de adopción de innovaciones de las TIC para apoyar actividades de aprovisionamiento.

\section{E. Variable preparación de proveedores y clien- tes en el uso de e-business}

Estudios anteriores sugieren la importancia que tiene para una empresa, la adecuada preparación tecnológica de sus socios comerciales (clientes y proveedores). Con relación a la adopción del negocio electrónico, autores como Jones et al. [12] o Zhu et al. [69] la consideran como una barrera importante y un aspecto determinante para la adopción del negocio electrónico, mientras que Chwelos et al. [64] creen que es un aspecto que está directamente relacionado con la intención de las empresas de usar aplicaciones EDI.

La utilidad de una innovación tecnológica aumenta en la medida que más empresas la adoptan [70], y la adopción requiere sistemas compatibles y una preparación tecnológica que facilite su implementación por parte de empresas y socios comerciales [39], [61]. Así pues, para obtener mejores beneficios y sacar más provecho del uso de las TIC en las relaciones con clientes y proveedores, es necesario que exista cierto nivel de homogeneidad en la preparación tecnológica de las empresas que buscan realizar negocios por vía electrónica. 
En consecuencia, la decisión de una empresa de adoptar las TIC para soportar su proceso de aprovisionamiento puede estar influenciada por el grado de preparación de sus socios comerciales actuales o potenciales [12], [28], [34], [35], [39], [61], [64], [66], [69], [70], preparación que no sólo se refiere a la infraestructura TIC disponible, sino a nivel de preparación de sus procesos y del personal para realizar actividades de negocio electrónico y compartir información comercial. Este aspecto, entonces, da origen a una nueva hipótesis:

Las empresas que consideran que no usan más intensivamente el negocio electrónico porque creen que sus proveedores y clientes no están preparados para ello, tienden a estar menos avanzadas en términos de adopción de innovaciones de las TIC para apoyar actividades de aprovisionamiento empresarial.

\section{METODOLOGÍA DE INVESTIGACIÓN}

\section{A. Fuente de datos}

Para la comprobación empírica del modelo se empleó la información recolectada por E-business W@tch, que es una iniciativa europea puesta en práctica y desarrollada por EMPIRICA -institución de investigación localizada en Bonn, Alemania-. En concreto, se ha empleado la encuesta sobre negocio electrónico e-business W@tch 2007 [71], la cual ofrece información relevante en relación al uso del negocio electrónico a nivel general, y del aprovisionamiento electrónico a nivel particular.

La base de datos e-business W@tch ha sido usada en versiones anteriores en investigaciones relacionadas con el tema de estudio [33], [48]. Así pues, habida cuenta que el uso de información secundaria se justifica por razones de naturaleza económica, metodológica, del entorno y de las empresas [72] y que la información contenida en esta base de datos ofrece información relevante en relación al uso del negocio electrónico a nivel general, y del aprovisionamiento electrónico a nivel particular, se decidió emplear esta base de datos para probar el modelo propuesto teóricamente.

No se ha tomado información más reciente dado que la encuesta dejó de realizarse en el año
2007 justamente ${ }^{2}$, sin embargo, dada la naturaleza del fenómeno que se pretende analizar y la robustez y seriedad de los datos recolectados, se considera viable el uso de estos datos con fines académicos.

La población de este trabajo se limita al sector del mueble en España y la constituyen todas aquellas empresas de diez o más empleados y menos de 250, que realizan su actividad principal en este sector. La encuesta se desarrolla a nivel empresarial, esto es, los datos se recogen centrándose en la empresa (en lugar de en sus establecimientos), definiéndose empresa como una organización de uno o más establecimientos que consta de una unidad legal [71]. La muestra de este estudio la constituyen 120 empresas de las cuales el 86,6\% (103 empresas) son pequeñas empresas y el $13,4 \%$ restante (16 empresas) son medianas. Los subsectores $\mathrm{CNAE}^{3}$ estudiados, según la clasificación de 1993 vigente en 2007 son: 36.12 (fabricación de muebles de oficina y establecimientos comerciales), 36.13 (fabricación de muebles de cocina y baño) y 36.14 (fabricación de otros muebles) [71].

El trabajo de campo lo llevó a cabo la empresa IPSOS, y las encuestas se realizaron de forma telefónica, mediante tecnología asistida por ordenador (Computer-Aided Telephone Interviewing - CATI). La persona a la que iba dirigido el cuestionario era normalmente el responsable de las TIC, y para las empresas que no contaban con este cargo, se entrevistó al gerente, y cada entrevista se realizó en un promedio de 20 minutos 24 segundos [71].

Estructuralmente esta encuesta se divide en siete módulos que sistematizan la información específica en relación a la adopción de las TIC en el negocio electrónico y específicamente en actividades de aprovisionamiento electrónico. Las preguntas seleccionadas para el estudio, fueron tanto aquellas específicas de e-procurement como las que, por su carácter genérico, pueden referirse al negocio electrónico con proveedores,

2 El observatorio sectorial e-Business Watch funcionó en el marco de un contrato entre la Comisión Europea, la Dirección General de Industria y Comercio y Empirica $\mathrm{GmbH}$ y estuvo en funcionamiento hasta el año 2007. http://ec.europa.eu/enterprise/ archives/e-business-watch/about/sectoral ebiz.htm

3 Clasificación Nacional de Actividades Económicas en España - CNAE. www.ine.es 
y a partir de ellas se definieron las variables que conforman el modelo. En cuanto a la escala de medida de estas variables seleccionadas, son categóricas especialmente dicotómicas.

\section{B. Análisis estadístico}

El análisis del modelo teórico requiere la comprobación de hipótesis relacionales, es decir, hipótesis que enuncian una posible relación entre dos o más variables y su intensidad pero sin especificar la dirección de la relación [73], por lo que se hace indispensable recurrir a métodos estadísticos que permitan identificar esta relación. En el caso particular, habida cuenta que las variables disponibles son cualitativas de naturaleza binaria, para analizar el comportamiento de una variable con respecto a otra se acudió al uso de tablas de contingencia, que son tabulaciones cruzadas de dos variables categóricas o no métricas en las que las entradas son las frecuencias de respuesta que caen en cada celda de la matriz [74].

En consecuencia, uno de los test aplicados en este caso fue el $V$ de Cramer, que es una medida de asociación basada en la chi-cuadrado, que puede ser aplicado a Tablas de contingencia de cualquier tamaño y adopta valores entre 0 -para el caso de inexistencia de relación- y 1 -para cuando si existe relación- [75].

Ya que las variables son no métricas, no paramétricas, se puede recurrir también al análisis de correlaciones de Spearman, adecuado para variables de tipo nominal y ordinal [75]. El coeficiente de correlación Spearman $\left(r_{s}\right)$, puede variar de -1 (asociación o relación negativa perfecta) a +1 (asociación o relación positiva perfecta) pasando por 0 (no relación). Tanto -1 como +1 son igualmente fuertes pero con interpretaciones opuestas desde altamente negativa hasta altamente positiva [76].

Dadas las limitaciones asociadas al hecho que la información empírica no fue recolectada específicamente para analizar la adopción de innovaciones de las TIC en el aprovisionamiento empresarial, sino el negocio electrónico en general, se identificó la pertinencia de cada una de las preguntas en relación a las variables del modelo y se excluyeron aquellas que, por no estar relacionadas, no servían para medir ninguna variable.
En cuanto a la variable dependiente, la pertenencia a cada uno de los tres niveles de adopción dependía de si las empresas contaban con tecnologías como identificación por radio frecuencia, si realizaba pedidos en línea, si compartían información con proveedores, si contaban con tecnologías de intercambio electrónico de datos con proveedores y el tipo de formato empleado.

En definitiva, el análisis bivariado se realizó entre la variable dependiente Nivel de adopción de innovaciones TIC para el aprovisionamiento electrónico[1], y las variables independientes: Ubicación de los proveedores, Presión de los proveedores y Preparación de proveedores y clientes en el uso del e-business, que dieron como resultado los coeficientes de asociación $V$ de Cramer y los coeficientes de correlación Ro de Spearman que se detallan en la Tabla 1 y en la Fig. 2.

TABLA I.

COEFICIENTES DE CORRELACIÓN ENTRE VARIABLES

\begin{tabular}{|l|l|l|}
\hline & \multicolumn{2}{|l|}{$\begin{array}{l}\text { Adopción de innovaciones TIC } \\
\text { en el aprovisionamiento empre- } \\
\text { sarial }\end{array}$} \\
\hline & Ro de Spearman & V de Cramer \\
\hline $\begin{array}{l}\text { E2_Ubicación de } \\
\text { proveedores }\end{array}$ & $.197 *$ & .177 \\
\hline $\begin{array}{l}\text { E3_Presión de pro- } \\
\text { veedores }\end{array}$ & $.233 *$ & $.264 * *$ \\
\hline $\begin{array}{l}\text { E4_Preparación_pro- } \\
\text { veedores_clientes }\end{array}$ & .99 & .168 \\
\hline $\begin{array}{l}\text { ** La correlación es significativa al nivel 0,01 } \\
\text { * La correlación es significativa al nivel 0,05 }\end{array}$ \\
\hline
\end{tabular}

Fuente: Autor del proyecto con base en datos procesados en SPSS V19

Fig. 2. RELACIÓN CON EL NIVEL DE ADOPCIÓN DE INNOVACIONES TIC PARA EL APROVISIONAMIENTO EMPRESARIAL



* * La correlación es significativa al nivel 0,01

* La correlación es significativa al nivel 0,05

Fuente: Autor del proyecto

A partir de la información presentada en la Tabla 1 y Figura. 2, es posible identificar que sólo se encontró asociación de las variables "Presión de los proveedores" y "Ubicación geográfica de pro- 
veedores" con la variable dependiente. Es decir, no hay evidencia estadística que permita afirmar que la preparación de proveedores y clientes en el uso de las TIC para apoyar procesos de negocio estén relacionados con el nivel de adopción de innovaciones TIC en el aprovisionamiento empresarial, contrario a lo que sucede con la presión ejercida y la ubicación de proveedores para la adopción de innovaciones TIC para soportar procesos de negocio, variables con la cuales si se evidencia relación.

\section{DISCUSIÓN DE RESULTADOS}

Es necesario recordar que el estudio se basó en los resultados de la encuesta realizada por ebusiness W@tch para el sector del mueble en España, estudio que si bien no fue efectuado específicamente con el objetivo de analizar el proceso de adopción de innovaciones de las TIC en actividades de aprovisionamiento empresarial, sí incluye información relevante del proceso de adopción del negocio electrónico a nivel general. Esta condición permite, por medio de una investigación exploratoria, hacer una primera identificación del conjunto de variables externas con potencial explicativo del fenómeno de adopción de innovaciones de las TIC en el aprovisionamiento empresarial y poder así obtener información para llevar a cabo una investigación más completa en el contexto particular.

En cuanto a las características generales de la muestra, el $86,6 \%$ son pequeñas (entre 10 y 49 empleados) y $13,4 \%$ medianas (entre 50 y 249 empleados), en su mayoría enfocadas al mercado nacional y sólo un $5 \%$ de empresas se enfoca principalmente al mercado de exportación.

Con respecto a la variable que mide la ubicación geográfica de proveedores, esta variable no está soportada teóricamente en el sentido estricto, sino que es una variable adaptada del "efecto de la ubicación del mercado" [39], [61], [62]. La relación encontrada en este con el Ro de Spearman es estadísticamente significativa al 5\%, y permite suponer que así como la ubicación geográfica de clientes está relacionada con la adopción del comercio electrónico, también la ubicación geográfica de proveedores puede estar relacionada con la adopción de innovaciones TIC para el aprovisionamiento, lo cual tiene sentido tenidas en cuenta las posibilidades que ofrecen las TIC para acercar a clientes y proveedores en un mismo entorno virtual, en que las distancias geográficas ya no son una limitante. Si bien para una variable categórica no tiene sentido hablar de relación positiva o negativa, por las características de esta variable el resultado puede significar que a mayor distancia geográfica con los proveedores mayor tendencia a adoptar las TIC para el aprovisionamiento empresarial.

El segundo aspecto analizado es la presión externa ejercida por los proveedores para que las empresas adopten determinadas TIC. Esta variable mostró relación estadísticamente significativa con la variable dependiente (tanto con el $V$ de Cramer como con el Ro de Spearman), lo que indica que las empresas que han sentido algún tipo de presión externa suelen estar en niveles superiores de adopción de las TIC. Este resultado es coherente con investigaciones anteriores en la adopción de e-procurement [31] o de sistemas EDI [29], [64], [65], y respalda la afirmación de que los procesos de cooperación y coordinación entre partners requieren del uso de tecnologías de información para potenciar sus resultados, lo que conlleva a que las empresas recomienden su uso a sus partners [14].

En este sentido, es probable que los proveedores de alguna manera puedan, si no presionar, al menos motivar a las empresas a usar tecnologías que faciliten y soporten sus comunicaciones, más aún si los proveedores involucrados cuentan con poder de negociación importante.

En todo caso, hay que tener en cuenta de una parte que sólo 3 empresas afirman expresamente haber recibido este tipo de presión de sus proveedores, y de otra parte que las materias primas del sector del mueble (textiles, colas, pinturas, barnices y en su mayoría tableros y madera), no son inputs estandarizados ni usualmente comercializadas por medios electrónicos, por lo que para posibles generalizaciones este resultado debe tomarse con cautela.

El último aspecto analizado fue el grado de preparación de socios comerciales para la adopción de innovaciones TIC para el aprovisionamiento, el cual no resultó tener relación estadísticamente significativa con la variable dependiente. Es decir, las empresas que consideran que no usan más intensivamente el negocio electrónico porque creen 
que los socios comerciales no están preparados para ello, no necesariamente tienden a estar más avanzadas en términos de adopción de innovaciones de TIC para apoyar actividades de aprovisionamiento empresarial.

Teóricamente la preparación de socios comerciales es un aspecto determinante en la adopción de las TIC tal como se evidencia en diversas investigaciones [12], [28], [34], [35], [39], [61], [64], [66], [69], [70], ya que requiere acciones conducentes a facilitar el intercambio automatizado de información, sin embargo, el hecho de no evidenciarse relación puede estar asociado a que la percepción de falta de preparación de clientes es generalizada, debido a la poca difusión de las TIC en el sector del mueble, así como a las características propias de las materias primas que compran las empresas o la complejidad de la categorización y la codificación industrial [71], lo que conlleva a que las empresas prefieran realizar sus compras por métodos tradicionales.

Estos resultados fueron presentados a expertos de la industria del mueble en España [77], [78] quienes afirman que no es extraño este comportamiento dado que aunque las empresas cuenten con herramientas tecnológicas usan de manera intensiva el papel como medio de comunicación.

Una posible explicación a este fenómeno puede ser que las empresas no adoptan las TIC porque no lo consideran necesario, no conocen sus beneficios o no se sienten preparadas para usarlas, creando un bucle del que sólo se puede salir gracias a las decididas inversiones de las empresas pioneras. Este resultado está en la misma línea del realizado por Hernández-Ortega y Serrano-Cinca [44], que consideran que las empresas no están dispuestas a adoptar las TIC a menos que su entorno así lo demande, mientras que el resto de agentes del entorno argumenta la misma justificación.

Este limitado uso de las TIC en los procesos de compra ya se ha identificado en trabajos anteriores con empresas de diferentes sectores, que encuentran evidencias empíricas que les permite afirmar de una parte que no son precisamente las materias primas las que suelen comprarse por medios electrónicos, sino otro tipo de insumos no productivos, y de otra parte que si bien las empresas pueden contar con tecnología e-procurement, ésta se emplea más para intercambiar informa- ción de cualquier tipo con proveedores que para realizar compras electrónicas [41]. Adicionalmente, con respecto al sector del mueble en España, se ha comprobado que aunque las empresas cuenten con herramientas TIC básicas para comunicarse con proveedores, éstas no se utilizan y en su lugar se emplean soportes en papel [77], por lo que no es de extrañar que el uso de las TIC no esté suficientemente generalizado en el sector, lo que ha condicionado su adopción a lo largo de toda la cadena de suministro, y las empresas que han incorporado las TIC no necesariamente hacen uso estratégico de ellas [65].

Pese a lo anterior, vale la pena resaltar que, dadas las limitaciones de la encuesta, el grado de preparación de clientes y proveedores para adoptar TIC en procesos de negocio se mide a través de la percepción subjetiva de la persona que responde la encuesta, por lo que los resultados deben tomarse con cautela.

Finalmente, vale la pena resaltar que si bien el modelo se analizó con base en datos empíricos del año 2007, dado que las variables analizadas no están en función del tiempo, se considera que los resultados son válidos para conocer la situación particular del sector en un momento específico. Adicionalmente, la robustez y calidad de los datos permitió probar de manera adecuada el modelo, lo que se constituye en un aporte valioso a la teoría de adopción de innovaciones TIC, que abren importantes oportunidades para la realización de futuros estudios que permitan replicar el modelo en otros sectores y en condiciones diferentes de la economía.

\section{CONCLUSIONES}

Como resultado del presente estudio, el cual pretendía examinar los factores del entorno empresarial, asociados con la adopción de innovaciones TIC para el aprovisionamiento empresarial en pequeñas y medianas empresas, se encontró en primer lugar, relación estadísticamente significativa entre el nivel de adopciones de las TIC en actividades de aprovisionamiento empresarial y la presión externa ejercida por los proveedores así como con su ubicación geográfica.

La relación identificada con la presión externa de proveedores sugiere que empresas que han sentido algún tipo de presión externa suelen estar 
en niveles superiores de adopción de innovaciones TIC de e-procurement. Este resultado pueda dar origen a futuros estudios que permitan comprobar el nivel de influencia de los proveedores con poder de negociación importante.

La relación encontrada con la ubicación geográfica de los proveedores permite suponer que así como la ubicación geográfica de clientes puede llegar a afectar la adopción de comercio electrónico, también la ubicación geográfica de proveedores puede estar relacionada con la adopción de innovaciones TIC para soportar el aprovisionamiento empresarial, lo cual tiene sentido según las posibilidades que ofrecen las TIC para acercar a clientes y proveedores en un mismo entorno virtual, en que las distancias geográficas ya no son una limitante

No se encontraron evidencias empíricas que permitan identificar posible relación con la preparación de clientes y proveedores para el nivel de adopción de innovaciones TIC en el aprovisionamiento empresarial. Esta falta de evidencia relacional puede ser debida a aspectos como las características de las empresas del sector y la forma como se relacionan a lo largo de la cadena de suministro, la penetración de las TIC en el sector del mueble o la dinámica propia de las TIC, que ofrecen, cada vez, mayor facilidad de acceso por parte de las Pymes no sólo en cuestión de coste, sino de confiabilidad, sencillez y posibilidades ofrecidas. Así como a factores metodológicos como las características de las preguntas empleadas, la subjetividad de las respuestas asociada a la percepción de quién responde o la selección de la muestra entre otros posibles.

Finalmente, el artículo presenta un modelo teórico validado para un grupo de empresas de un sector específico y en un momento determinado en el tiempo, razón por la cual se considera valiosa la realización de nuevos estudios que permitan de una parte confirmar las relaciones encontradas con el nivel de adopción de innovaciones TIC en el aprovisionamiento empresarial, y de otra parte demostrar si efectivamente hay o no relación entre las variables cuya relación no se pudo comprobar, o incluso si existen otros aspectos que no fueron considerados en el presente estudio y que pueden complementar el modelo para el contexto empresarial específico.

\section{REFERENCIAS}

[1] A. S. Aguilar-Jiménez, Las tecnologías de información en pymes manufactureras. Procesos de adopción y factores de influencia. Primera. Editorial Académica Española, 2011.

[2] D. Servera y I. Gil, «Tecnologías de la información y la comunicación en la gestión logística», Distribución y Consumo, No. 98, pp. 67-82, 2008.

[3] J. M. Torrecilla, «Las estrategias operativas de la empresa. El tiempo como factor competitivo», Economía Industrial, n. ${ }^{\circ}$ 330, 1999.

[4] L. De-Boer, J. Harink, y G. Heijboer, «A Conceptual Model for Assessing the Impact of Electronic Procurement», European Journal of purchasing \& supply management, No. 8, pp. 25-33, 2000.

[5] J. R. De La Torre, M. Herias, F. San Nicolás, y P. Herrera, "Las Pymes y el mercado digital", Navarra, 2001, pp. 153-176.

[6] M. Attaran, "The Coming Age of Online Procurement", Industrial Management \& Data System, vol. 101, No. 4, pp. 177-180, 2001.

[7] B. Heywood, M. Barton, y C. Heywood, e-Procurement. Managing Successful E-procurement Implementation. London: Financial Times. Prentice Hall. Pearson Education Limited, 2002.

[8] B. A. Wagner, I. Fillis, y U. Jhohansson, "E-Business and E-supply Strategy in Small and Medium Sized Business (SMEs)", Supply Chain Management: An International Journal, Vol. 8, No. 4, pp. 343-354, 2003.

[9] S. R. Croom y A. Brandon-Jones, "Impact of E-procurement: Experiences from Implementation in the UK Public Sector", Journal of purchasing \& supply chain management, №. 13, pp. 294-303, 2007.

[10] F. Hernández, J. Tafur, y M. Palacios, "Sistema e-procurement: propuesta de un business case como soporte de toma de decisión. E-procurement system: a business case proposal to support decision making", en XII Congreso de Ingeniería de Organización. 2nd International Conference on Industrial Engineering and Industrial Management, 2008.

[11] I. Diéguez Castrillón, A. I. Gueimonde, y A. I. Sinde, "Gestión de recursos humanos, organización del trabajo y nuevas tecnologías: sus vínculos con los resultados.", Valencia, 2006.

[12] P. Jones, P. Beynon-Davies, y E. Muir, «Ebusiness Barriers to Growth Within the SME Sectorn, Journal of Systems \& Information Technology, vol. 7, No. 1, pp. 1-26, 2003.

[13] J. Hartley, M. Lane, y E. Duplaga, «Exploring the Barriers to the Adoption of E-auctions for Sourcing", Inter- 
national Journal of operations \& production management, vol. 26, No. 2, pp. 202-221, 2006.

[14] T. Teo, S. Lin, y K. Lai, "Adopters and Non-adopters of E-procurement in Singapore: An empirical study", Omega - The International Journal of Management Science, No. 37, pp. 972-987, 2009.

[15] P. Chau, «Inhibitors to EDI Adoption in Small Business: An Empirical Investigation", Journal of Electronic Commerce Research, Vol. 2, No. 2, pp. 78-88, 2001.

[16] M. Frohlich, «E-Integration in the Supply Chain: Barriers and Performance", Decision Sciences, vol. 33, No. 4, pp. 537-556, 2002.

[17] A. Pranato, J. McKay, y P. Marshall, «Exploring the Perceptions of Inhibitors and Drivers of E-business Progression Among SMEs at Different Stages of E-business Maturity", European Conference on Information Systems, 2004.

[18] J. M. Láñez, "Los marketplaces en España", iWorld, 01jul-2002. .

[19] C. Gibson y R. Nolan, "Managing the Four Stages of EDP Growth", Harvard Business Review, vol. 76, No. 88, 1974.

[20] R. Nolan, D. C. Croson, y K. Seger, "The Stages Theory: A Framework for IT adoption and Organizational Learning”. Harvard Business School, 19-mar-1993.

[21] A. Pranato, J. McKay, y P. Marshall, "Frameworks to Support E-business Growth Strategy", Slovenia, 2001, pp. $1254-1263$.

[22] J. J. Anaya y S. Polanco, Innovación y mejora de procesos logísticos. Análisis, diagnóstico e implantación de sistemas logísticos. Madrid: ESIC - Téciman, 2005.

[23] M. Calvo y Z. González, "Análisis de las tecnologías de información que emplean las pymes", en in Modelos de Rasch en Administración de Empresas, Santa Cruz de Tenerife: FYDE-Caja Canarias, 2006, pp. 38-48.

[24] B. Ramdani y P. Kawaiek, "SME Adoption of Enterprise Systems in the Northwest of England: An Environmental, Technological, and Organizational Perspective", en in IFIP International Federation for Information Processing, Manchester: Organizational Dynamics of Technology-Based Innovation: Diversifying the Research Agenda, 2007, pp. 409-430.

[25] E. Rogers, Diffusion of Innovations, vol. Quinta edición. Free Press, 2003.

[26] R. Nolan, "Information Technology Management from 1960-2000", Harvard Business School, 2001.

[27] T. Teo y Y. Pian, "A Model for Web Adoption", Information \& Management, vol. 41, pp. 457-468, 2004.
[28] J. M. Cepeda Pérez y M. Á. Plaza Mejía, "Aspectos interorganizacionales de intercambio electrónico de documentos", Revista Dirección y Organización, No. 31, pp. 68-80, feb. 2005.

[29] C. lacovou, I. Benbasat, y A. Dexter, «Electronic Data Interchange and Small Organizations: Adoption and Impact of Technology", MIS Quarterly, vol. 19, No. 4, pp. 465-485, dic. 1995.

[30] R. Heckman, "Organizing and Managing Supplier Relationships in Information Technology Procurement", International Journal of Information Management, vol. 19, pp. 141-155, 1999.

[31] J. K. Chan y M. K. Lee, "SME E-Procurement Adoption in Hong Kong - The Roles of Power, Trust and Value", Hong Kong, 2003.

[32] A. Ordanini, Information Technology and Small Businesses. Antecedents and Consequences of Technology Adoption. Edward Elgar Publishing Limeted, 2006.

[33] R. Batenburg, "E-procurement Adoption by European Firms: A Quantitative Analysis", Journal of Purchasing \& Supply Management, No. 13, pp. 182-192, 2007.

[34] E. Prier y C. McCue, "E-procurement Adoption in Local Governments of the United States", Government Procurement, pp. 12-31, feb. 2007.

[35] F. Wu, G. A. Zsidisin, y A. D. Ross, "Antecedents and Outcomes of E-Procurement Adoption: An Integrative Model", IEEE Transactions on Engineering Management, vol. 54, n. ${ }^{\circ}$ 3, pp. 576-587, 2007.

[36] A. Gunasekaran y E. Ngai, "Adoption of E-procurement in Hong Kong: An Empirical Research", International Journal of Production Economics, n. ${ }^{\circ} 113$, pp. 159175, 2008.

[37] K. Arbin, "The Structure of Determinants of Individual Adoption and Use of E-ordering Systems", Human Systems Management, n. ${ }^{\circ}$ 27, pp. 143-159, 2008.

[38] M. Rahim, «Identifying Factors Affecting Acceptance of E-procurement Systems: An Initial Qualitative Study at an Australian City Council", 10th International-Business-Information-Management-Association Conference, vol. 3, pp. 219-229, 2008.

[39] A. Soares-Aguiar y A. Palma-dos-Reis, "Why do firms adopt E-Procurement Systems? Using Logistic Regression to Empirical Test a Conceptual Model", IEEE Transactions on Engineering Management, vol. 55, n. ${ }^{\circ}$ 1, feb. 2008.

[40] G. Vaidyanathan y S. Devaraj, "The Role of Quality in E-procurement Performance: An Empirical Analysis", Journal of Operations Management, No. 26, pp. 407425, 2008. 
[41] A. Gunasekaran, R. McGaughey, E. Ngai, y B. Rai, "EProcurement Adoption in the Southcoast SMEs", International Journal of Production Economics, No. 122, pp. 161-175, 2009.

[42] B. Hernández-Ortega, J. Jiménez-Martínez, y M. J. Martín-DeHoyos, "The Effect of Experience on Web Procurement. An Intersectorial Analysis of Small Firms", International Journal of Entrepreneurial Behaviour \& Research, vol. 15, No. 1, pp. 7-24, 2009.

[43] M. Kaliannan, M. Raman, y M. Dorasamy, «E-Procurement Adoption in the Malaysian Public Sector: Organizational Perspectives", en Enterprise Distributed Object Computing Conference Workshops, EDOCW 2009.13th, 2009, pp. 189-194.

[44] B. Hernández-Ortega y C. Serrano-Cinca, "Qué induce a las empresas a adoptar facturación electrónica? Efecto de las percepciones y del entorno competitivo", Universia Business review, n. ${ }^{\circ}$ 24, pp. 96-121, 2009.

[45] R. Amit y C. Zott, "Value Creation in E-Business", Strategic Management Journal,, vol. 22, pp. 493-529, 2001.

[46] K. Zhu, K. Kraemer, S. Xu, y J. Dedrick, «Information Technology Payoff in E-business Environments: An International Perspective on Value Creation of E-business in the Financial Services Industry", Journal of Management Information Systems, vol. 21, No. 1, pp. 17-54, 2004.

[47] K. Zhu y K. Kraemer, "Post-Adoption Variations in Usage and Value of E-business by Organizations: Cross-Country Evidence From the Retail Industry", Information Systems Research, vol. 16, No. 1, pp. 61-84, mar. 2005.

[48] P. Soto-Acosta, «Análisis de la creación de valor en el negocio electrónico a partir de la teoría de recursos y capacidades", Tesis Doctoral. Universidad de Murcia., Murcia, 2006.

[49] E. Grandon y M. Pearson, "Electronic Commerce Adoption: An Empirical Study of Small and Medium US Business", Information \& Management, vol. 42, pp. 197216, abr. 2004.

[50] S. Pires y L. E. Carretero, Gestión de la cadena de suministros. Mc Graw Hill, 2007.

[51] R. Lancioni, M. Smith, y T. Oliva, "The rol of the Internet in Supply Chain Management", Industrial Marketing Management, n. ${ }^{\circ}$ 29, pp. 45-56, 2000.

[52] A. Cater-Steel, "Steps to Internet Adoption Success: A Study of Four Small Regional Organisations", Australasian Journal of Information Systems, vol. 11, No.2, may 2004.

[53] F. Alonso y G. Fitzgerald, "Theoretical Approaches to Study SMEs eBusiness Progression", Journal of Computing and Information Technology - CIT 13, vol. 2, pp. 123136, 2005.
[54] A. Constanzo A., "El comercio B2B cada vez más presente en las Pyme", N-Economía, Madrid, jul. 2008.

[55] S. Nambisan y Y.-M. Wang, "Readblocks to Web Technology Adoption?", Communications of the ACM, vol. 42, n. ${ }^{\circ}$, ene. 1999.

[56] E. Daniel, H. Wilson, y A. Myer, «Adoption of E-Commerce by SMEs in the UK. Towards a Stage Model", International Small Business Journal, vol. 20, No. 3, pp. 253-270, 2002.

[57] J. G. Cegarra Navarro, J. Alonso Martínez, y A. F. Monreal Garres, "Influencia del uso de las tecnologías de internet en el capital estructural", Investigaciones Europeas de Dirección y Economía de la Empresa, vol. 12, No. 3, pp. 217-228, 2006.

[58] L. Tornatzky y M. Fleischer, The Processes of Technological Innovation. Lexington Books, 1990.

[59] T. Ravichandran y C. Lertwongsatien, "Effect of Information Systems Resources and Capabilities on Firm Performance: A Resource-Based Perspective", Journal of Management Information Systems, vol. 21, No.4, pp. 237-276, 2005.

[60] K. Kuan y P. Chau, "A Perception-Based Model for EDI Adoption in Small Business Using a TechnologyOganization-Environment Framework", Information \& Management, No. 38, pp. 507-521, 2001.

[61] E. Gide y M. Wu, "A Study of E-commerce Business Satisfaction Model to Measure E-commerce Success in Service SMEs", International Journal of Electronic Customer Relationship Management, vol. 1, No. 3, pp. 307-325, 2007.

[62] L. Kaewkitipong y D. Brown, "Adoption and Evaluation of E-business in Thai SMEs: A Process Perspective", en Americas Conference on Information Systems (AMCIS), 2008

[63] H. Hollenstein y M. W"rter, "The Decision to Adopt Internet-Based E-commerce. An Empirical Analysis Based on Swiss Firm-Level Data", en 15TH Biennial Conference of the international telecommunications society (ITS) - Connecting societies and markets: Communication technology, policy and impacts, 2004.

[64] P. Chwelos, I. Benbasat, y A. Dexter, «Empirical Test of an EDI Adoption Model", Information Systems Research, vol. 12, No. 3, pp. 304-321, 2001.

[65] D. L. González-Bañales, M. G. Leyva Alanís, y J. A. Gutiérrez Reyes, "Datamining aplicado para el diagnóstico de la incorporación las TIC en las micro y pequeñas empresas", presentado en IV Congreso Iberoamericano de Soporte al Conocimiento con la tecnología. Las $\mathrm{TIC}$, herramienta clave para la innovación y el crecimiento sostenible, Bucaramanga, Colombia, 2012. 
[66] J. Mehrtens, P. Cragg, y A. Mills, «A Model of Internet Adoption by SMEs", Information \& Management, No. 39, pp. 165-176, 2001

[67] M. Caldeira y J. Ward, "Using Resource-Based Theory to Interpret the Successful Adoption and Use of Information Systems \& Technology in Manufacturing Small and Medium Sized Enterprises", en Global Co-Operation in the New Millennium - The 9th European Conference on Information Systems, 2001.

[68] P. Chau y K.-L. Hui, "Determinants of Small Business EDI Adoption: An Empirical Investigation", Journal of Organizational Computing and Electronic Commerce, vol. 11,No. 4, pp. 229-252, 2001.

[69] K. Zhu, K. Kraemer, y S. Xu, «A Cross-Country Study of Electronic Business Adoption Using the Technology-Organization-Environment Framework", en Twenty-third International Conference on Information Systems, 2002.

[70] G. Premkumar, "A Meta-Analysis of Research on Information Technology Implementation in Small Business", Journal of Organizational Computing and Electronic Commerce, vol. 13, No. 2, pp. 91-121, 2003.

[71] European Commission, «e-Business W@tch Survey 2007. Diseño y Metodología”. 2008.
[72] I. Grande Esteban, "Fuentes de información para la investigación. Las bases de datos", en in Metodología para la investigación en marketing y dirección de empresas, Madrid: Pirámide, 1999, pp. 95-123.

[73] F. Sarabia, "Definición del tema a investigar", en in Metodología de la investigación en dirección de empresas, Madrid: Pirámide, 1999, pp. 69-94.

[74] J. J. Hair, R. Anderson, R. Tatham, y W. Black, Análisis multivariante, vol. 5. Madrid: Pearson, Prentice Hall, 2004.

[75] S. Miquel, E. Bigné, J.-P. Lévy, A. Cuenca, y M. J. Miquel, Investigación de Mercados. Madrid: Mc Graw Hill, 1997.

[76] G. Morgan, N. Leech, G. Gloeckner, y K. Barrett, SPSS for Introductory Statistics, Use and Interpretation, vol. second. London: Lawrence Erlbaum Associates Publishers, 2004.

[77] J. Solana, Entrevista personal. Resultados del proyecto de Modelado de Redes de Empresas del Centre de Difusió Tecnológica de la Fusta i el Moble de Catalunya. 2009.

[78] M. J. Núñez, Entrevista personal. Desarrollos TIC en el sector de la madera y el mueble en Valencia - AIDIMA. 2010. 